\title{
The Calculation of Fourier Integrals
}

\section{By Guy de Balbine and Joel N. Franklin}

1. Introduction. The numerical calculation of Fourier integrals

$$
\int_{-\infty}^{\infty} f(x) e^{i \omega x} d x \quad(-\infty<\omega<\infty)
$$

is difficult for two reasons: (i) the range of integration is infinite $(-\infty<x<\infty)$; (ii) the integrand oscillates rapidly for large $\omega$.

A complicated but effective method of numerical integration has been developed by Hurwitz and Zweifel [1]. We will show that this method is equivalent to a certain trapezoidal rule. We will show that an Euler transformation approximates the Fourier integral by infinite series which are convergent and which are asymptotic for large $\omega$.

2. The Method of Hurwitz and Zweifel. If we write $f(x)$ as the sum of an even function and an odd function

$$
\frac{1}{2}[f(x)+f(-x)]=\frac{1}{2} \psi(x), \frac{1}{2}[f(x)-f(-x)]=\frac{1}{2} \phi(x),
$$

the integral (1.1) takes the form $C(\omega)+i S(\omega)$, where

$$
C(\omega)=\int_{0}^{\infty} \psi(x) \cos \omega x d x, \quad S(\omega)=\int_{0}^{\infty} \phi(x) \sin \omega x d x .
$$

In these integrals we make, respectively, the changes of variable

$$
x=(\pi / \omega) y, \quad x=(\pi / \omega)\left(y+\frac{1}{2}\right) .
$$

Then the integrals take the forms

$$
\begin{aligned}
& C(\omega)=\frac{\pi}{2 \omega} \int_{-\infty}^{\infty} \psi\left(\frac{\pi}{\omega} y\right) \cos \pi y d y \\
& S(\omega)=\frac{\pi}{2 \omega} \int_{-\infty}^{\infty} \phi\left(\frac{\pi}{\omega}\left(y+\frac{1}{2}\right)\right) \cos \pi y d y .
\end{aligned}
$$

Using the transformation

$$
\int_{-\infty}^{\infty} \chi(y) d y=\int_{-1 / 2}^{1 / 2} \sum_{n=-\infty}^{\infty} \chi(y+n) d y
$$

which is valid if summation and integration may be interchanged, we find from (2.4)

$$
\begin{aligned}
& C(\omega)=\frac{\pi}{2 \omega} \int_{-1 / 2}^{1 / 2} \gamma(y, \omega) \cos \pi y d y \\
& S(\omega)=\frac{\pi}{2 \omega} \int_{-1 / 2}^{1 / 2} \sigma(y, \omega) \cos \pi y d y,
\end{aligned}
$$

Received March 16, 1966. 
where

$$
\begin{aligned}
& \gamma(y, \omega)=\sum_{n=-\infty}^{\infty}(-)^{n} \psi\left(\frac{\pi}{\omega}(y+n)\right) ; \\
& \sigma(y, \omega)=\sum_{n=-\infty}^{\infty}(-)^{n} \phi\left(\frac{\pi}{\omega}\left(y+n+\frac{1}{2}\right)\right) .
\end{aligned}
$$

As functions of $y$ both $\gamma$ and $\sigma$ satisfy the identities

$$
F(y) \equiv F(-y) ; \quad F(y+1) \equiv-F(y) ; \quad F\left(\frac{1}{2}+y\right) \equiv-F\left(\frac{1}{2}-y\right) .
$$

A function $F(y)$ with the properties (2.7) has period 2. If $F(y)$ has a convergent Fourier series

$$
F(y)=\sum_{k=0}^{\infty}[() \cos k \pi y+() \sin k \pi y]
$$

then only the terms $\cos k \pi y$, with $k$ odd, have nonzero coefficients. Thus, $\gamma(y, \omega)$ and $\sigma(y, \omega)$ have Fourier series of the form

$$
\sum_{\nu=0}^{\infty}() \cos (2 \nu+1) \pi y
$$

where the coefficients ( ) are functions of $\omega$.

Hurwitz and Zweifel now develop a variant of Gaussian quadrature which is exact when $\gamma(y, \omega)$ or $\sigma(y, \omega)$ is replaced in (2.5) by the truncated Fourier series

$$
\sum_{\nu=0}^{2 N-1} a_{\nu}(\omega) \cos (2 \nu+1) \pi y .
$$

Every $\cos (2 \nu+1) \pi y$ is an odd polynomial in $\cos \pi y$ :

$$
\cos (2 \nu+1) \pi y=\sum_{\mu=0}^{\nu}()(\cos \pi y)^{2 \mu+1} .
$$

Therefore, if a series (2.9) is multiplied by $\cos \pi y$, the result may be expressed in the form

$$
\cos ^{2} \pi y \sum_{\nu=0}^{2 N-1} \alpha_{\nu}(\omega)\left(\cos ^{2} \pi y\right)^{\nu}
$$

Thus, we must look for the rule of Gaussian quadrature which is exact for integrals of the form

$$
I(\omega)=\int_{0}^{1 / 2} \cos ^{2} \pi y \sum_{\nu=0}^{2 N-1} \alpha_{\nu}(\omega)\left(\cos ^{2} \pi y\right)^{\nu} d y .
$$

Let $u=\cos ^{2} \pi y$. We require orthogonal polynomials $\Gamma_{0}(u), \Gamma_{1}(u), \cdots, \Gamma_{N}(u)$ satisfying

$$
\int_{0}^{1 / 2} u \Gamma_{j}(u) \Gamma_{k}(u) d y=0 \text { for } j \neq k .
$$

[Hurwitz and Zweifel use the notation $\Gamma_{j}(\cos \pi y)$ instead of our $\Gamma_{j}(u)=$ $\Gamma_{j}\left(\cos ^{2} \pi y\right)$.] Explicitly, we have 


$$
\Gamma_{k}(u)=\frac{\cos (2 k+1) \pi y}{\cos \pi y}=\frac{T_{2 k+1}\left(u^{1 / 2}\right)}{u^{1 / 2}}
$$

where $T_{n}$ is the Tchebicheff polynomial of degree $n$. Let $u_{1}>u_{2}>\cdots>u_{N}$ be the zeros of $\Gamma_{N}(u)$; explicitly,

$$
u_{j}=\cos ^{2} \pi y_{j} \quad \text { with } y_{j}=(2 j-1) /(2 N+1) 2 \quad(j=1, \cdots, N) .
$$

Let $f(u)$ be any polynomial of the form

$$
f(u)=\sum_{\nu=0}^{2 N-1} \alpha_{\nu} u^{\nu}
$$

as in (2.11). Let $f(u)$ be interpolated at $u_{1}, \cdots, u_{N}$ by a polynomial $p(u)$ of degree $\leqq N-1$ :

$$
f\left(u_{j}\right)=p\left(u_{j}\right) \quad(j=1, \cdots, N) .
$$

Then

$$
\int_{0}^{1 / 2} u f(u) d y=\int_{0}^{1 / 2} u p(u) d y
$$

because

$$
f(u)-p(u)=\Gamma_{N}(u) q(u),
$$

where the quotient $q(u)$ has degree $\leqq N-1$, so that

$$
\int_{0}^{1 / 2} u \Gamma_{N}(u) q(u) d y=0
$$

By the Lagrange interpolation formula,

$$
p(u)=\sum_{j=1}^{N} \pi_{j}(\grave{u}) f\left(u_{j}\right)
$$

where $\pi_{j}(u)$ is the polynomial of degree $N-1$ satisfying $\pi_{j}\left(u_{k}\right)=\delta_{j k}$; the polynomials $\pi_{j}(u)$ are independent of the function $f(u)$. The identity (2.17) now yields

$$
\int_{0}^{1 / 2} u f(u) d y=\sum_{j=1}^{N} c_{j} f\left(u_{j}\right)
$$

where $c_{1}, \cdots, c_{N}$ are the Christoffel numbers

$$
c_{j}=\int_{0}^{1 / 2} u \pi_{j}(u) d y \quad(j=1, \cdots, N) .
$$

The Christoffel numbers are independent of $f(u)$. The identity (2.19) holds for all polynomials $f(u)$ of degree $\leqq 2 N-1$ in the variable $u=\cos ^{2} \pi y$.

Hurwitz and Zweifel suggest that the Christoffel numbers [which they call $\left.W_{j}{ }^{(N)}\right]$ be determined by setting $f(u) \equiv u^{\nu-1}(\nu=1, \cdots, N)$ in the identity (2.19). The resulting system of equations

$$
\int_{0}^{1 / 2} u^{\nu} d y=\sum_{j=1}^{N}\left(u_{j}\right)^{\nu-1} c_{j} \quad(\nu=1, \cdots, N)
$$


determines the $c_{j}$ uniquely because

$$
\operatorname{det}\left(u_{j}^{\nu-1}\right)_{j, \nu=1, \cdots, N}=\prod_{j>k}\left(u_{j}-u_{k}\right) \neq 0 .
$$

The left-hand side of (2.21) has the explicit value

$$
\int_{0}^{1 / 2} \cos ^{2 \nu} \pi y d y=\frac{1 \cdot 3 \cdot 5 \cdots(2 \nu-1)}{2^{\nu+1}(\nu !)} \quad(\nu=1, \cdots, N),
$$

and the numbers $u_{j}$ were given in (2.14). In their paper [1] Hurwitz and Zweifel recommend that the $c_{j}=W_{j}{ }^{(N)}$ be evaluated numerically for any given $N$.

The final quadrature formulas for the integrals $C(\omega)$ and $S(\omega)$ are

$$
C_{N}(\omega)=\frac{\pi}{\omega} \sum_{j=1}^{N} c_{j} \frac{\gamma\left(y_{j}, \omega\right)}{\cos \pi y_{j}} ; \quad S_{N}(\omega)=\frac{\pi}{\omega} \sum_{j=1}^{N} c_{j} \frac{\sigma\left(y_{j}, \omega\right)}{\cos \pi y_{j}} .
$$

We have $C_{N}(\omega)=C(\omega)$ and $S_{N}(\omega)=S(\omega)$ when $\gamma$ and $\sigma$ have finite Fourier series of the form

$$
\sum_{\nu=0}^{2 N-1} a_{\nu}(\omega) \cos (2 \nu+1) \pi y
$$

3. Simplification of the Hurwitz-Zweifel Method. First we will show that the Christoffel numbers $c_{j}=W_{j}{ }^{(N)}$ have the values

$$
c_{j}=(2 N+1)^{-1} \cos ^{2} \pi y_{j}
$$

$$
(j=1, \cdots, N)
$$

where $y_{j}=(2 j-1) / 2(2 N+1)$.

Proof. Define the functions

$$
p_{\nu-1}(u)=\frac{\cos 2 \nu \pi y+(-1)^{\nu+1}}{\cos ^{2} \pi y} \quad(\nu=1, \cdots, N) .
$$

We assert that $p_{\nu-1}(u)$ is a polynomial of degree $\nu-1$ in the variable $u=\cos ^{2} \pi y$. This is true because the numerator of (3.2) is a polynomial in $u$ of degree $\nu$. Further, the numerator vanishes when $u=0$, which occurs when $y=\frac{1}{2}$. Since the denominator of (3.2) is $u$, the fraction (3.2) is a polynomial of degree $\nu-1$.

To prove (3.1), it is sufficient to show that the $N$ values (3.1) satisfy the $N$ equations

$$
\int_{0}^{1 / 2} u p_{\nu-1}(u) d y=\sum_{j=1}^{N} p_{\nu-1}\left(u_{j}\right) c_{j} \quad(\nu=1, \cdots, N)
$$

because these equations are true if and only if the equations (2.21) hold. The lefthand side of (3.3) equals

$$
\int_{0}^{1 / 2}\left[\cos 2 \nu \pi y+(-1)^{\nu+1}\right] d y=\frac{1}{2}(-1)^{\nu+1} \quad(\nu=1, \cdots, N) .
$$

Substitution of (3.1) and (3.2) in the right-hand side of (3.3) yields

$$
\sum_{j=1}^{N} p_{\nu-1}\left(u_{j}\right) c_{j}=\sum_{j=1}^{N}\left[\cos 2 \nu \pi y_{j}+(-1)^{\nu+1}\right](2 N+1)^{-1} \text {. }
$$


Let $\beta=\nu \pi /(2 N+1)$. Then, since $0<\beta<\pi / 2$,

$$
\begin{aligned}
\sum_{j=1}^{N} \cos 2 \nu \pi y_{j} & =\operatorname{Re} \sum_{j=1}^{N} \exp (2 j-1) \beta i \\
& =\operatorname{Re} e^{\beta i} \sum_{j=0}^{N-1}\left(e^{2 \beta i}\right)^{j} \\
& =\operatorname{Re} e^{\beta i}\left(e^{2 N \beta i}-1\right) /\left(e^{2 \beta i}-1\right) \\
& =(\sin 2 N \beta) /(2 \sin \beta) .
\end{aligned}
$$

But

$$
\sin 2 N \beta=\sin (2 N \nu \pi /(2 N+1))=\sin (\nu \pi-\beta)=(-)^{\nu+1} \sin \beta .
$$

Therefore, the sum (3.5) equals

$$
(-1)^{\nu+1}\left[\frac{1}{2}+N\right](2 N+1)^{-1}=\frac{1}{2}(-1)^{\nu+1} .
$$

Since the value (3.5) for the right-hand side of (3.3) equals the value (3.4) for the left-hand side, formula (3.1) is now proved.

Theorem 3.1. Let $\Delta y=1 / p$, where $p$ is any positive integer. For any function $f(y)$ define the trapezoidal sum

$$
T[\Delta y ; f(y)]=\Delta y\left[\frac{1}{2} f\left(-\frac{1}{2}\right)+\sum_{\nu=1}^{p-1} f\left(-\frac{1}{2}+\nu \Delta y\right)+\frac{1}{2} f\left(\frac{1}{2}\right)\right] .
$$

Let $C_{N}(\omega)$ and $S_{N}(\omega)$ be the Gaussian quadrature sums (2.24). Then

$$
\begin{aligned}
& C_{N}(\omega)=(\pi / 2 \omega) T\left[(2 N+1)^{-1} ; \gamma(y, \omega) \cos \pi y\right] ; \\
& S_{N}(\omega)=(\pi / 2 \omega) T\left[(2 N+1)^{-1} ; \sigma(y, \omega) \cos \pi y\right] .
\end{aligned}
$$

Proof. Set $\Delta y=1 /(2 N+1)$. By (3.1) and (2.24),

$$
C_{N}(\omega)=\frac{\pi}{\omega}(\Delta y) \sum_{j=1}^{N} \gamma\left(y_{j}, \omega\right) \cos \pi y_{j}
$$

where $y_{j}=\left(j-\frac{1}{2}\right) \Delta y$. Since $\gamma(y, \omega)$ is an even function of $y$, we have

$$
C_{N}(\omega)=\frac{\pi}{2 \omega}(\Delta y) \sum_{\nu=1}^{2 N} \gamma\left(-\frac{1}{2}+\nu \Delta y, \omega\right) \cos \pi\left(-\frac{1}{2}+\nu \Delta y\right)
$$

because

$$
-\frac{1}{2}+\nu \Delta y= \pm y_{j} \text { for } \nu=N+j \text { and } \nu=N-j+1 .
$$

The sum (3.8) equals the first trapezoidal sum (3.6), except that the terms involving $f\left( \pm \frac{1}{2}\right)$ are missing. But these terms equal zero when $f(y)=\gamma(y, \omega) \cos \pi y$. This proves the first identity (3.6). The second identity is established by the same reasoning applied to $f(y)=\sigma(y, \omega) \cos \pi y$.

We will now express the Gaussian sums $C_{N}(\omega), S_{N}(\omega)$ in terms of the functions $\psi(x), \phi(x)$ which appear in the original Fourier integrals $(2.2)$. We will show that $C_{N}(\omega)$ and $S_{N}(\omega)$ are directly expressible by means of the simplest of all approximations to a Fourier integral

$$
\int_{-\infty}^{\infty} f(x) \exp (-i \omega x) d x
$$


namely

$$
F\left[x_{0}, \Delta x, \omega ; f(x)\right]=\Delta x \sum_{\nu=-\infty}^{\infty} f\left(x_{0}+\nu \Delta x\right) \exp \left(-i \omega\left(x_{0}+\nu \Delta x\right)\right) .
$$

Theorem 3.2. Let $C_{N}(\omega), S_{N}(\omega)$ be the Gaussian sums (2.24). Let $\gamma(y, \omega)$ and $\sigma(y, \omega)$ be defined for real $\omega \neq 0$ by convergent series (2.6). Then

$$
\begin{aligned}
& C_{N}(\omega)=\frac{1}{2} F[\pi / 2(2 N+1) \omega, \pi /(2 N+1) \omega, \omega ; \psi(x)] ; \\
& S_{N}(\omega)=(i / 2) F[0, \pi /(2 N+1) \omega, \omega ; \phi(x)] .
\end{aligned}
$$

Proof. Let $\Delta x=\pi /[(2 N+1) \omega]=(\pi / \omega) \Delta y$. By (3.8),

$$
C_{N}(\omega)=\frac{1}{2} \Delta x \sum_{\nu=0}^{2 N} \gamma\left(-\frac{1}{2}+\nu \Delta y, \omega\right) \cos \pi\left(-\frac{1}{2}+\nu \Delta y\right) .
$$

In this sum we may use the lower limit $\nu=0$ instead of $\nu=1$ because the term with $\nu=0$ equals zero. From (2.6) we have

$$
C_{N}(\omega)=\frac{1}{2} \Delta x \sum_{\nu=0}^{2 N} \sum_{n=-\infty}^{\infty}(-)^{n} \psi\left(\frac{\pi}{\omega}\left(-\frac{1}{2}+\nu \Delta y+n\right)\right) \cos \pi\left(-\frac{1}{2}+\nu \Delta y\right) .
$$

Since $\Delta y=1 /(2 N+1)$, and since $(-)^{n} \cos \pi \theta=\cos \pi(\theta+n)$,

$$
C_{N}(\omega)=\frac{1}{2} \Delta x \sum_{\nu=-\infty}^{\infty} \psi\left(\frac{\pi}{\omega}\left(-\frac{1}{2}+\nu \Delta y\right)\right) \cos \pi\left(-\frac{1}{2}+\nu \Delta y\right) .
$$

Set $\nu=N+1+\mu(-\infty<\mu<\infty)$. Then

$$
\frac{\pi}{\omega}\left(-\frac{1}{2}+\nu \Delta y\right)=\left(\frac{\pi}{\omega}\right) \frac{\mu+\frac{1}{2}}{2 N+1}=\left(\mu+\frac{1}{2}\right) \Delta x .
$$

Therefore,

$$
C_{N}(\omega)=\frac{1}{2} \Delta x \sum_{\mu=-\infty}^{\infty} \psi\left(\left(\mu+\frac{1}{2}\right) \Delta x\right) \cos \omega\left(\mu+\frac{1}{2}\right) \Delta x
$$

Since $\psi(x)=\psi(-x)$, we have

$$
\psi\left(\left(\mu+\frac{1}{2}\right) \Delta x\right) \sin \omega\left(\mu+\frac{1}{2}\right) \Delta x=-\psi\left(\left(\mu^{\prime}+\frac{1}{2}\right) \Delta x\right) \sin \omega\left(\mu^{\prime}+\frac{1}{2}\right) \Delta x
$$

when $\mu^{\prime}=-\mu-1(\mu=0,1, \cdots)$. Therefore, $\cos \omega\left(\mu+\frac{1}{2}\right) \Delta x$ may be replaced by $\exp \left[-i \omega\left(\mu+\frac{1}{2}\right) \Delta x\right]$ in the sum (3.14). This proves the first identity (3.10).

For $S_{N}(\omega)$ we have, corresponding to $(3.11)$, the formula

$$
S_{N}(\omega)=\frac{1}{2} \Delta x \sum_{\nu=0}^{2 N} \sigma\left(-\frac{1}{2}+\nu \Delta y, \omega\right) \cos \pi\left(-\frac{1}{2}+\nu \Delta y\right) .
$$

The series (2.6) for $\sigma(y, \omega)$ now yields

$$
S_{N}(\omega)=\frac{1}{2} \Delta x \sum_{\nu=0}^{2 N} \sum_{n=-\infty}^{\infty}(-)^{n} \phi\left(\frac{\pi}{\omega}(\nu \Delta y+n)\right) \sin \pi \nu \Delta y .
$$

Since $(-)^{n} \sin \pi \theta=\sin \pi(\theta+n)$, and since $\Delta y=1 /(2 N+1)$,

$$
S_{N}(\omega)=\frac{1}{2} \Delta x \sum_{\nu=-\infty}^{\infty} \phi(\nu \Delta x) \sin \omega \nu \Delta x
$$


Because $\phi(x)=\phi(-x)$, sin $\omega \nu \Delta x$ may be replaced by $\exp (-i \omega \nu \Delta x)$. This completes the proof.

Corollary 3.1. We have, equivalent to (3.10), the identities

$$
\begin{aligned}
& C_{N}(\omega)=\Delta x \sum_{\mu=0}^{\infty} \psi\left(\left(\mu+\frac{1}{2}\right) \Delta x\right) \cos \omega\left(\mu+\frac{1}{2}\right) \Delta x ; \\
& S_{N}(\omega)=\Delta x \sum_{\nu=1}^{\infty} \phi(\nu \Delta x) \sin \omega \nu \Delta x,
\end{aligned}
$$

where $\Delta x=\pi /[(2 N+1) \omega]$.

Proof. These identities are restatements of (3.14) and (3.17).

4. Estimation of the Remainder. In the preceding section we showed that the method of Hurwitz and Zweifel is equivalent to the use of an infinite trapezoidal sum $F\left[x_{0}, \Delta x, \omega ; f(x)\right]$ to approximate the Fourier integral

$$
F[\omega ; f(x)]=\int_{-\infty}^{\infty} f(x) e^{-i \omega x} d x .
$$

Given $f(x)$, we wish to estimate the remainder

$$
R\left[x_{0}, \Delta x, \omega ; f(x)\right]=F\left[x_{0}, \Delta x, \omega ; f(x)\right]-F[\omega ; f(x)] .
$$

We wish also to examine different rules of numerical integration, for example, Simpson's rule

$$
F_{s}\left[x_{0}, \Delta x, \omega ; f(x)\right]=\frac{2 \Delta x}{3}\left(\sum_{\nu \text { even }}+2 \sum_{\nu \text { odd }}\right) f\left(x_{0}+\nu \Delta x\right) \exp \left(-i \omega\left(x_{0}+\nu \Delta x\right)\right) .
$$

The usual local-error formulas for the trapezoidal rule and for Simpson's rule are (see [5], p. 73)

$$
\begin{aligned}
\frac{\Delta x}{2}[g(a)+g(a+\Delta x)] & \\
=\int_{a}^{a+\Delta x} g(x) d x+\frac{(\Delta x)^{3}}{12} g^{\prime \prime}(\xi) & (a \leqq \xi \leqq a+\Delta x), \\
\frac{\Delta x}{6}[g(a)+4 g(a+\Delta x)+g(a+2 \Delta x)] & \\
=\int_{a}^{a+2 \Delta x} g(x) d x+\frac{(\Delta x)^{5}}{90} g^{(4)}\left(\xi_{s}\right) & \left(a \leqq \xi_{s} \leqq a+2 \Delta x\right) .
\end{aligned}
$$

These formulas suggest this conclusion: For small $\Delta x$ Simpson's rule must be two orders of magnitude more accurate than the trapezoidal rule. A simple consideration of symmetry will show that this conclusion is false when the formulas (4.4) and (4.5) are applied an infinite number of times to approximate an infinite-range integral $\int_{-\infty}^{\infty} g(x) d x$. In our context the function $g(x)$ has the particular form $g(x)=$ $f(x) \exp (-i \omega x)$, but that is irrelevant. Using the definition (4.3), define the error in Simpson's rule:

$$
R_{s}\left[x_{0}, \Delta x, \omega ; f(x)\right]=F_{s}\left[x_{0}, \Delta x, \omega ; f(x)\right]-F[\omega ; f(x)] .
$$


From the definitions of $F$ and $F_{s}$, we have

$$
F\left[x_{0}, \Delta x, \omega ; f(x)\right]=\frac{1}{2} F_{s}\left[x_{0}, \Delta x, \omega ; f(x)\right]+\frac{1}{2} F_{s}\left[x_{0}+\Delta x, \Delta x, \omega ; f(x)\right] .
$$

Therefore,

$$
R\left[x_{0}, \Delta x, \omega ; f(x)\right]=\frac{1}{2} R_{s}\left[x_{0}, \Delta x, \omega ; f(x)\right]+\frac{1}{2} R_{s}\left[x_{0}+\Delta x, \Delta x, \omega ; f(x)\right] .
$$

Thus, the remainder in the trapezoidal rule is the arithmetic mean of two remainders for Simpson's rule with the same $\Delta x$. Therefore, the trapezoidal rule is at least as accurate as Simpson's rule for infinite-range integrals. Remarks of this nature have been made by Y. L. Luke [8].

A simple generalization shows that the trapezoidal rule is at least as good as any other rule of quadrature for infinite-range integrals. Here we assume equally spaced abscissae. Let

$$
G_{c}\left[x_{0}, \Delta x ; g(x)\right]=(\Delta x) \sum_{\nu=-\infty}^{\infty} c_{\nu} g\left(x_{0}+\nu \Delta x\right)
$$

be any sum approximating integrals $\int_{-\infty}^{\infty} g(x) d x$. Assume that the coefficients $c_{\nu}$ are independent of $x_{0}, \Delta x$, and $g(x)$, and suppose that the coefficients are periodic: $c_{\nu} \equiv c_{\nu+p}$. For Simpson's rule,

$$
c_{0}=\frac{2}{3}, \quad c_{1}=\frac{4}{3} ; \quad p=2 .
$$

We shall also require the consistency-condition

$$
c_{0}+c_{1}+\cdots+c_{p-1}=p .
$$

Let

$$
G\left[x_{0}, \Delta x ; g(x)\right]=\Delta x \sum_{\nu=-\infty}^{\infty} g\left(x_{0}+\nu \Delta x\right)
$$

and define the associated remainders

$$
R_{c}\left[x_{0}, \Delta x ; g\right]=G_{c}\left[x_{0}, \Delta x ; g\right]-\int_{-\infty}^{\infty} g(x) d x ; \quad R=G-\int_{-\infty}^{\infty} .
$$

Then the periodicity and consistency of the coefficients imply

$$
R\left[x_{0}, \Delta x ; g\right]=\frac{1}{p} \sum_{\mu=0}^{p-1} R_{c}\left[x_{0}+\mu \Delta x, \Delta x ; g\right] .
$$

Thus, as $\Delta x \rightarrow 0, R$ goes to zero at least as fast as the remainders $R_{c}$.

One may also write $R_{c}$ in terms of $R$ :

$$
R_{c}\left[x_{0}, \Delta x ; g\right]=\sum_{\mu=0}^{p-1} c_{\mu} R\left[x_{0}+\mu \Delta x, p \Delta x ; g\right] .
$$

The increment $\Delta x$ in the remainder $R_{c}$ is replaced by the larger increment $p \Delta x$ $(p \geqq 2)$ in the remainders $R$. Thus, from the identity (4.14) we may not conclude that, as $\Delta x \rightarrow 0, R_{c}$ goes to zero at least as fast as the remainders $R$.

To obtain a useful form for the remainder (4.2) in the calculation of Fourier integrals, we shall express the remainder in terms of the unknown Fourier integral 
$F[\omega ; f]$. To do this we will use the Poisson summation formula. Let $h(x)$ be defined as a function of bounded variation for $-\infty<x<\infty$. Let the infinite series

$$
\sum_{\nu=-\infty}^{\infty} h(\nu)
$$

be convergent. If any integer $\nu$ is a point of discontinuity of $h(x)$, assume that $h(\nu)$ is the arithmetic mean of the limits $h(\nu \pm 0)$ from right and left; more stringently, assume that the function

$$
x^{-1}[h(\nu+x)+h(\nu-x)-2 h(\nu)]
$$

is Lebesgue-integrable in the neighborhood of $x=0$. (This will surely be true if

$$
h(\nu)=\frac{1}{2}[h(\nu+0)+h(\nu-0)]
$$

and if $h(x)$ has derivatives $h^{\prime}(\nu \pm 0)$ from the right and from the left at $x=\nu$.) Assume that the Fourier integral

$$
H(\lambda)=\int_{-\infty}^{\infty} h(x) e^{-i \lambda x} d x
$$

converges for all $\omega$ which are multiples of $2 \pi$. Then a well-known theorem of Poisson states:

$$
\sum_{\nu=-\infty}^{\infty} h(\nu)=\lim _{M \rightarrow \infty} \sum_{|m| \leqq M} H(2 \pi m) .
$$

To obtain $F\left[x_{0}, \Delta x, \omega ; f\right]$, as defined by (3.9), set

$$
h(x)=(\Delta x) f\left(x_{0}+x \Delta x\right) \exp \left(-i \omega\left(x_{0}+x \Delta x\right)\right) .
$$

The Fourier transform (4.17) equals

$$
H(\lambda)=\int_{-\infty}^{\infty}(\Delta x) f\left(x_{0}+x \Delta x\right) \exp \left(-i \omega\left(x_{0}+x \Delta x\right)\right) e^{-i \lambda x} d x .
$$

The change of variable $\xi=x_{0}+x \Delta x$ gives

$$
H(\lambda)=\exp \left(i \lambda x_{0} / \Delta x\right) \int_{-\infty}^{\infty} f(\xi) \exp (-i(\omega+\lambda / \Delta x) \xi) d \xi .
$$

In the notation (4.1), this expression equals

$$
H(\lambda)=\exp \left(i \lambda x_{0} / \Delta x\right) F[\omega+\lambda / \Delta x ; f] .
$$

The theorem of Poisson now yields

$$
\begin{aligned}
F\left[x_{0}, \Delta x, \omega ; f\right] & =\lim _{M \rightarrow \infty} \sum_{|m| \leqq M} \exp \left(\frac{i 2 m \pi x_{0}}{\Delta x}\right) F\left[\omega+\frac{2 m \pi}{\Delta x} ; f\right] \\
& =F[\omega ; f]+R\left[x_{0}, \Delta x, \omega ; f\right]
\end{aligned}
$$

where, if $\beta=2 \pi x_{0} / \Delta x$,

$$
R\left[x_{0}, \Delta x, \omega ; f\right]=\sum_{m=1}^{\infty}\left\{e^{i m \beta} F\left[\omega+\frac{2 m \pi}{\Delta x} ; f\right]+e^{-i m \beta} F\left[\omega-\frac{2 m \pi}{\Delta x} ; f\right]\right\} .
$$


We have just proved the following theorem:

THEOREM 4.1. Let $f(x)$ be of bounded variation for $-\infty<x<\infty$. If a is any point of discontinuity of $f$, assume that $[f(a+x)+f(a-x)-2 f(a)] / x$ is Lebesgue integrable in the neighborhood of $x=0$. Suppose that the Fourier integral

$$
F[\omega ; f]=\int_{-\infty}^{\infty} f(x) e^{-i \omega x} d x
$$

converges for all real $\omega$. Let the infinite-series

$$
F\left[x_{0}, \Delta x, \omega ; f\right]=\Delta x \sum_{\nu=-\infty}^{\infty} f\left(x_{0}+{ }_{\mu} \Delta x\right) \exp \left(-i \omega_{i}\left(x_{0}+\nu \Delta x\right)\right)
$$

converge for some fixed $x_{0}, \Delta x>0$, and $\omega$. Then

$$
F\left[x_{0}, \Delta x, \omega ; f\right]=F[\omega, f]+R\left[x_{0}, \Delta x, \omega ; f\right],
$$

where $R$ is the convergent series (4.23).

We can immediately apply this theorem to obtain an expression for the remainder when the series $C_{N}(\omega)$ of Hurwitz and Zweifel is used to approximate the cosine-integral $C(\omega)$, or when $S_{N}(\omega)$ is used to approximate the sine-integral $S(\omega)$. From (3.10) we find

$$
C_{N}(\omega)-C(\omega)=\frac{1}{2} R\left[x_{0}, \Delta x, \omega ; \psi(x)\right]
$$

with

$$
x_{0}=\frac{1}{2} \Delta x, \quad \Delta x=\pi /(2 N+1) \omega .
$$

Since $\psi(x)$ is even, we have

$$
\frac{1}{2} F[\omega ; \psi(x)]=C(\omega)=C(-\omega) .
$$

Therefore, by (4.27) and (4.23),

$$
C_{N}(\omega)-C(\omega)=\sum_{m=1}^{\infty}(-)^{m}\{C([2 m(2 N+1)+1] \omega)
$$

$$
+C([2 m(2 N+1)-1] \omega)\} .
$$

For the sine-integral we have

$$
S(\omega)=\int_{0}^{\infty} \phi(x) \sin \omega x d x=\frac{i}{2} F[\omega ; \phi(x)]=-S(-\omega) .
$$

Now (3.10) and (4.23) give

$$
\begin{aligned}
S_{N}(\omega)-S(\omega) & =(i / 2) R[0, \pi /(2 N+1) \omega, \omega ; \phi(x)] \\
S_{N}(\omega)-S(\omega)= & \sum_{m=1}^{\infty}\{S([2 m(2 N+1)+1] \omega) \\
& -S([2 m(2 N+1)-1] \omega)\} .
\end{aligned}
$$

The remainders for other rules of quadrature can be found at once from the identity (4.14). For example, in Simpson's rule we have 


$$
\begin{aligned}
& G_{c}\left[x_{0}, \Delta x ; f(x) e^{-i \omega x}\right] \\
& =(\Delta x)\left(\frac{1}{3} \sum_{\nu \text { even }}+\frac{2}{3} \sum_{\nu \text { odd }}\right) f\left(x_{0}+\nu \Delta x\right) \exp \left(-i \omega\left(x_{0}+\nu \Delta x\right)\right) \\
& =\int_{-\infty}^{\infty} f(x) e^{-i \omega x} d x+R_{c}\left[x_{0}, \Delta x ; f(x) e^{-i \omega x}\right]
\end{aligned}
$$

where

$$
\begin{aligned}
R_{c}\left[x_{0}, \Delta x ; f(x) e^{-i \omega x}\right]= & \frac{1}{3} R\left[x_{0}, 2 \Delta x ; f(x) e^{-i \omega x}\right] \\
& +\frac{2}{3} R\left[x_{0}+\Delta x, 2 \Delta x ; f(x) e^{-i \omega x}\right] .
\end{aligned}
$$

But $R\left[x_{0}, \Delta x ; f(x) e^{-i \omega x}\right] \equiv R\left[x_{0}, \Delta x, \omega ; f\right]$. Therefore, by (4.23), the remainder in Simpson's rule is

$$
\begin{aligned}
R_{c}\left[x_{0}, \Delta x ; f(x) e^{-i \omega x}\right] & \\
=\sum_{m=1}^{\infty} & \left\{\exp \left(\frac{i m \pi x_{0}}{\Delta x}\right)\left(\frac{1}{3}+(-1)^{m} \frac{2}{3}\right) F\left[\omega+\frac{m \pi}{\Delta x} ; f\right]\right. \\
& \left.+\exp \left(-i m \pi x_{0} / \Delta x\right)\left(\frac{1}{3}+(-1)^{m} \frac{2}{3}\right) F\left[\omega-\frac{m \pi}{\Delta x} ; f\right]\right\} .
\end{aligned}
$$

A comparison of the remainders (4.23) and (4.32) shows a superiority of the trapezoidal rule. Suppose that the function $f(x)$ is band-limited, as in many engineering applications. Assume, for some $\Omega>0$,

$$
F[\omega ; f] \equiv 0 \text { for } \omega>\Omega .
$$

Let $-\Omega<\omega<\Omega$. Formula (4.32) shows that Simpson's rule is exact, i.e. the remainder is zero, if

$$
\omega-\pi / \Delta x<-\Omega \text { and } \omega+\pi / \Delta x>\Omega
$$

which is the case if

$$
\Delta x<\pi /(|\omega|+\Omega)
$$

But (4.23) shows that the trapezoidal rule is exact if

$$
\Delta x<2 \pi /(|\omega|+\Omega) .
$$

Thus, $\Delta x$ may be taken twice as large in the trapezoidal rule. The next theorem states that the tolerance (4.35) uniquely characterizes the trapezoidal rule.

Theorem 4.2. Let $c_{\nu}(\nu=0, \pm 1, \pm 2, \cdots)$ be absolute constants. Let $\Omega>0$. Let $B(\Omega, \mathbf{c})$ be the class of functions $f(x)$ for which the series

$$
\Delta x \sum_{\nu=-\infty}^{\infty} c_{r} f\left(x_{0}+\nu \Delta x\right) \exp \left(-i \omega\left(x_{0}+\nu \Delta x\right)\right)
$$

converges when $x_{0}$ is real and $\Delta x>0$ and $-\Omega<\omega<\Omega$; and for which the Fourier transform

$$
F(\omega)=\int_{-\infty}^{\infty} f(x) e^{-i \omega x} d x
$$


is piecewise continuous and piecewise continuously differentiable for $-\Omega<\omega<\Omega$, with $F(\omega) \equiv 0$ for $|\omega|>\Omega$. Assume that, when $-\Omega<\omega<\Omega$, the convergent series (4.36) equals the Fourier integral $F(\omega)$ for all $f(x)$ in the class of band-limited functions $B(\Omega, \mathrm{c})$ for all $\Delta x<2 \pi /(|\omega|+\Omega)$. Then all $c_{\nu}=1$.

Proof. Given the coefficients $c_{\nu}(\nu=0, \pm 1, \cdots)$ and the frequency-limit $\Omega>0$, define

$$
f(x)=\sin \Omega\left(x-x_{0}-j \Delta x\right) / \pi\left(x-x_{0}-j \Delta x\right)
$$

where $j$ is an integer and where $\Delta x=\pi / \Omega$. This function has the Fourier transform

$$
\begin{array}{lrl}
F(\omega) & =\exp \left(-i \omega\left(x_{0}+j \Delta x\right)\right) & (-\Omega<\omega<\Omega) \\
F(\omega) \equiv 0 & (|\omega|>\Omega) .
\end{array}
$$

Further, for $\nu=0, \pm 1, \cdots$

$$
\begin{aligned}
& f\left(x_{0}+\nu \Delta x\right)=0 \quad \text { if } \quad \nu \neq j, \\
& f\left(x_{0}+j \Delta x\right)=\Omega / \pi .
\end{aligned}
$$

Therefore, $f(x)$ lies in the class $B(\Omega, \mathbf{c})$.

The infinite series (4.36) has the value

$$
(\Delta x) c_{j}(\Omega / \pi) \exp \left(-i \omega\left(x_{0}+j \Delta x\right)\right)=c_{j} \exp \left(-i \omega\left(x_{0}+j \Delta x\right)\right) .
$$

Since

$$
\Delta x=\pi / \Omega<2 \pi /(|\omega|+\Omega) \text { when }-\Omega<\omega<\Omega
$$

the hypothesis of the theorem requires that the series-value (4.40) equal the Fourier-integral value:

$$
c_{j} \exp \left(-i \omega\left(x_{0}+j \Delta x\right)\right)=F(\omega)=\exp \left(-i \omega\left(x_{0}+j \Delta x\right)\right) \quad(-\Omega<\omega<\Omega) .
$$

Therefore, $c_{j}=1$. Since $j$ can be any integer, the theorem is proved. The next theorem is a converse.

Theorem 4.3. Let $\Omega>0$. Let $B(\Omega)$ be the class of continuous functions $f(x)$ for which the series

$$
\Delta x \sum_{\nu=-\infty}^{\infty} f\left(x_{0}+\nu \Delta x\right) \exp \left(-i \omega\left(x_{0}+\nu \Delta x\right)\right)
$$

converges when $x_{0}$ is real and $\Delta x>0$ and $-\Omega<\omega<\Omega$; and for which the Fourier transform (4.37) exists and is square-integrable, with $F(\omega) \equiv 0$ for $|\omega|>\Omega$. Then, when $-\Omega<\omega<\Omega$, the convergent series (4.36.1) equals the Fourier integral $F(\omega)$ for all $f(x)$ in the class of band-limited functions $B(\Omega)$ for all $\Delta x<2 \pi /(|\omega|+\Omega)$.

Proof. We have proved this theorem, in the paragraph preceding Theorem 4.2, under the assumption (in Theorem 4.1) that $f(x)$ is of bounded variation for $-\infty<x<\infty$. We must deduce that the total variation $V$ of $f(x)$ is finite under the hypothesis of Theorem 4.3. For all $x$ we have

$$
f(x)=\frac{1}{2 \pi} \int_{-\Omega}^{\Omega} F(\omega) e^{i \omega x} d \omega
$$

The hypothesis that $f(x)$ is continuous is made to insure that the identity (4.41) holds for all $x$, and not merely except for a set of measure zero. 
The function (4.41) is continuously differentiable, and the total variation of $f(x)$ equals

$$
V=\int_{-\infty}^{\infty}\left|f^{\prime}(x)\right| d x \leqq+\infty .
$$

But

$$
\begin{aligned}
\left|f^{\prime}(x)\right| & =\left|\frac{1}{2 \pi} \int_{-\Omega}^{\Omega} F(\omega) i \omega e^{i \omega x} d \omega\right| \\
& \leqq \frac{1}{2 \pi} \int_{-\Omega}^{\Omega}|F(\omega) \omega| d \omega \equiv M<\infty .
\end{aligned}
$$

Therefore, by the hypothesis that $F(\omega)$ is square-integrable, and by Plancherel's Theorem,

$$
V \leqq \frac{1}{M} \int_{-\infty}^{\infty}\left|f^{\prime}(x)\right|^{2} d x=\frac{1}{2 \pi M} \int_{-\Omega}^{\Omega}|F(\omega)|^{2} \omega^{2} d \omega<\infty .
$$

This completes the proof that $V<\infty$.

The superiority of the trapezoidal rule is not restricted to band-limited functions. This point is shown in a general way by the identity (4.13), but we wish to present a specific example. Let

$$
f(x)=1 /\left(x^{2}+1\right) \quad(-\infty<x<\infty) .
$$

The Fourier transform is

$$
F[\omega ; f(x)]=\pi e^{-|\omega|} .
$$

By (4.23), the remainder in the trapezoidal rule is

$$
\begin{aligned}
R\left[x_{0}, \Delta x, \omega ; f\right]=\pi \sum_{m=1}^{\infty}\{ & \exp \left(i m 2 \pi x_{0} / \Delta x\right) \exp (-|\omega+2 m \pi / \Delta x|) \\
& \left.+\exp \left(-i m 2 \pi x_{0} / \Delta x\right) \cdot \exp (-|\omega-2 m \pi / \Delta x|)\right\} .
\end{aligned}
$$

As $\Delta x \rightarrow+0$, we have

$$
\begin{array}{r}
R\left[x_{0}, \Delta x, \omega ; f\right]=\left(\exp \left(2 \pi i x_{0} / \Delta x\right) e^{-\omega}+\exp \left(-2 \pi i x_{0} / \Delta x\right) e^{\omega}\right) \\
\cdot \exp (-2 \pi / \Delta x)+O(\exp (-4 \pi / \Delta x)) .
\end{array}
$$

To find the error in Simpson's rule with the same increment, $\Delta x$, we apply (4.14) with

$$
c_{0}=\frac{2}{3}, \quad c_{1}=\frac{4}{3} ; \quad p=2 ; \quad g(x)=f(x) e^{-i \omega x}=e^{-i \omega x} /\left(x^{2}+1\right) .
$$

Substitution of (4.46) in (4.14) now yields

$$
\begin{aligned}
R_{c}\left[x_{0}, \Delta x ; g\right]= & \frac{2}{3} R\left[x_{0}, 2 \Delta x ; g\right]+\frac{4}{3} R\left[x_{0}+\Delta x, 2 \Delta x ; g\right] \\
= & \frac{2}{3} R\left[x_{0}, 2 \Delta x, \omega ; f\right]+\frac{4}{3} R\left[x_{0}+\Delta x, 2 \Delta x, \omega ; f\right] \\
= & -\frac{2}{3}\left(\exp \left(\pi i x_{0} / \Delta x\right) e^{-\omega}+\exp \left(-\pi i x_{0} / \Delta x\right) e^{\omega}\right) \exp (-\pi / \Delta x) \\
& \quad+O(\exp (-2 \pi / \Delta x)) .
\end{aligned}
$$


Thus, as $\Delta x \rightarrow+0$ in this example, the error in Simpson's rule is much larger than the error in the trapezoidal rule.

5. The Convergent, Asymptotic Euler Series. Finally we wish to present a method for computing Fourier integrals which can be used for all nonzero frequencies.

For small $\omega$ or for large $\omega$ one could compute the Fourier integral in a straightforward way. For small $\omega$ the integrand oscillates very slowly, and there is no particular problem. An upper limit $x_{1}$ and an increment $\Delta x$ can be chosen so that (say for $S(\omega)$ )

$$
\int_{0}^{x_{1}} \sin \omega x \phi(x) d x
$$

is a good approximation to $S(\omega)$ for $0 \leqq \omega \leqq \omega_{0}$; and then the finite-range integral (5.1) could be computed, say, by Simpson's rule. If $\omega_{0}$ is small, the increment $\Delta x$ need not be very small.

For uniformly large $\omega$, there also is no great problem. There are asymptotic, divergent series which are very good numerically. For example, for $S(\omega)$ repeated integration by parts yields

$$
\int_{0}^{\infty} \sin \omega x \phi(x) d x \sim \frac{\phi(0)}{\omega}-\frac{\phi^{\prime \prime}(0)}{\omega^{3}}+\frac{\phi^{(4)}(0)}{\omega^{5}}-\cdots
$$

if all derivatives of $\phi$ are absolutely integrable. The example of $\phi(x)=1 /\left(1+x^{2}\right)$ illustrates that the asymptotic series (5.2) is usually divergent. This method, therefore, is useful only for large $\omega$. The series (5.2) has the added practical disadvantage of requiring analytic differentiation of the function $\phi(x)$, a process which is not easy to implement in a digital-computer subroutine for general usage. We will now show that a summation formula of Euler, applied to the trigonometric sums $C_{N}(\omega)$, $S_{N}(\omega)$, yields series which are asymptotic for large $\omega$ and convergent for all $\omega$. These series can be used for all $\omega$ varying between some small and large positive limits, $\omega_{0} \leqq \omega \leqq \omega_{1}$.

Theorem 5.1. Let $a_{0}-a_{1}+a_{2}-a_{3}+\cdots$ be a convergent series. Let

$$
\begin{gathered}
\delta a_{k}=a_{k}-a_{k+1}, \quad \delta^{2} a_{k}=a_{k}-2 a_{k+1}+a_{k+2}, \\
\delta^{3} a_{k}=a_{k}-3 a_{k+1}+3 a_{k+2}-a_{k+3}, \cdots .
\end{gathered}
$$

Then

$$
a_{0}-a_{1}+a_{2}-a_{3}+\cdots=\frac{1}{2} a_{0}+\frac{1}{4} \delta a_{0}+\frac{1}{8} \delta^{2} a_{0}+\frac{1}{16} \delta^{3} a_{0}+\cdots .
$$

Proof. This is Euler's transformation. The proof that the series on the right converges and equals $\sum(-)^{k} a_{k}$, provided only that the series $\sum(-)^{k} a_{k}$ converges, was first given in 1901 by L. D. Ames [7]. An exposition of the proof appears in Knopp [6, p. 245], to which we refer the reader.

For later reference, we note the result, in Knopp's exposition, that the remainder after $m$ terms of the Euler series is the convergent series

$$
R_{m}=\left(1 / 2^{m}\right)\left(\delta^{m} a_{0}-\delta^{m} a_{1}+\delta^{m} a_{2}-\delta^{m} a_{3}+\cdots\right) .
$$

The identity (5.4) states that $R_{m} \rightarrow 0$ as $m \rightarrow \infty$. 
To prove the asymptotic character of the Euler series, we shall later use this elementary result:

Lemma 5.1. For $x \geqq 0$ let $a(x)$ have continuous derivatives of order $\leqq m+1$. Let $R_{m}$ be a convergent series defined by (5.5), where $a_{k} \equiv a(k)$. Then

$$
\left|R_{m}\right| \leqq 2^{-m} \int_{0}^{\infty}\left|a^{(m+1)}(x)\right| d x
$$

if the infinite integral converges.

Proof. We first remark that

$$
\delta^{m} a_{k}=(-)^{m} \int_{0}^{1} \cdots \int_{0}^{1} a^{(m)}\left(k+u_{1}+\cdots+u_{m}\right) d u_{1} \cdots d u_{m} .
$$

This identity follows by induction: it is clearly true for $m=1$; and if it is true for any $m$, it implies

$$
\begin{aligned}
& \delta^{m+1} a_{k}=-\left(\delta^{m} a_{k+1}-\delta^{m} a_{k}\right) \\
&=(-)^{m+1} \int_{0}^{1} \cdots \int_{0}^{1}\left[a^{(m)}\left(k+1+u_{1}+\cdots+u_{m}\right)-a^{(m)}\left(k+u_{1}\right.\right. \\
&\left.\left.+\cdots+u_{m}\right)\right] d u_{1} \cdots d u_{m} \\
&=(-)^{m+1} \int_{0}^{1} \cdots \int_{0}^{1} a^{(m+1)}\left(k+u_{1}+\cdots+u_{m}+u_{m+1}\right) d u_{1} \\
& \cdots d u_{m} d u_{m+1}
\end{aligned}
$$

From (5.7) we have

$$
\begin{aligned}
\left|\delta^{m} a_{2 v}-\delta^{m} a_{2 v+1}\right| & \\
& =\mid \int_{0}^{1} \cdots \int_{0}^{1} \int_{2 v}^{2 v+1} a^{(m+1)}\left(y+u_{1}+\cdots+u_{m}\right) d y d u_{1} \cdots d u_{m} \\
& \leqq \int_{0}^{1} \cdots \int_{0}^{1} \int_{2 v}^{2 v+2}\left|a^{(m+1)}\left(y+u_{1}+\cdots+u_{m}\right)\right| d y d u_{1} \cdots d u_{m} .
\end{aligned}
$$

Summation of this inequality for $\nu=0, \cdots, n$ yields

$$
\begin{aligned}
\sum_{\nu=0}^{n} \mid \delta^{m} a_{2 \nu} & -\delta^{m} a_{2 \nu+1} \mid \\
& \leqq \int_{0}^{1} \cdots \int_{0}^{1} \int_{0}^{2 n+2}\left|a^{(m+1)}\left(y+u_{1}+\cdots+u_{m}\right)\right| d y d u_{1} \cdots d u_{m} \\
& \leqq \int_{0}^{1} \cdots \int_{0}^{1} \int_{0}^{\infty}\left|a^{(m+1)}\left(y+u_{1}+\cdots+u_{m}\right)\right| d y d u_{1} \cdots d u_{m}
\end{aligned}
$$

Let

$$
I(\xi)=\int_{\xi}^{\infty}\left|a^{(m+1)}(x)\right| d x
$$

Since the integrand is $\geqq 0$, we have $I(\xi) \leqq I(0)$. But the last expression $(5.10)$ equals the mean value 


$$
\int_{0}^{1} \cdots \int_{0}^{1} I\left(u_{1}+\cdots+u_{m}\right) d u_{1} \cdots d u_{m} \leqq I(0)=\int_{0}^{\infty}\left|a^{(m+1)}(x)\right| d x .
$$

To bound $\left|R_{m}\right|$, we now write

$$
\begin{aligned}
2^{-m} \sum_{k=0}^{2 n+1}(-)^{k} \delta^{m} a_{k} \mid & =\left|2^{-m} \sum_{\nu=0}^{n}\left(\delta^{m} a_{2 \nu}-\delta^{m} a_{2 v+1}\right)\right| \\
& \leqq 2^{-m} \sum_{\nu=0}^{n}\left|\delta^{m} a_{2 \nu}-\delta^{m} a_{2 v+1}\right| \leqq 2^{-m} \int_{0}^{\infty}\left|a^{(m+1)}(x)\right| d x
\end{aligned}
$$

Letting $n \rightarrow \infty$, we find the required inequality (5.6).

Lemma 5.2. Let $\omega>0$. Assume the convergence of the trapezoidal sums

$$
\begin{aligned}
& C_{N}(\omega)=\Delta x \sum_{\mu=0}^{\infty} \psi\left(\left(\mu+\frac{1}{2}\right) \Delta x\right) \cos \omega\left(\mu+\frac{1}{2}\right) \Delta x, \\
& S_{N}(\omega)=\Delta x \sum_{\nu=1}^{\infty} \phi(\nu \Delta x) \sin \omega \nu \Delta x,
\end{aligned}
$$

where $\Delta x=\pi /((2 N+1) \omega)$. Let $\psi(-x)=\psi(x)$. For $1 \leqq \nu \leqq 2 N$ let

$$
\begin{aligned}
& c(k)=c_{k}=\frac{1}{2} \psi\left((\pi / \omega)\left[\nu /(2 N+1)+k-\frac{1}{2}\right]\right), \\
& s(k)=s_{k}=\phi((\pi / \omega)(\nu /(2 N+1)+k)) \quad(k=0,1,2, \cdots) .
\end{aligned}
$$

Let $\delta c_{k}=c_{k}-c_{k+1}$. Then

$$
\begin{aligned}
& C_{N}(\omega)=\frac{\pi}{(2 N+1) \omega} \sum_{\nu=1}^{2 N}\left(\delta c_{0}-\delta c_{1}+\delta c_{2}-\delta c_{3}+\cdots\right) \sin \frac{\nu \pi}{2 N+1}, \\
& S_{N}(\omega)=\frac{\pi}{(2 N+1) \omega} \sum_{\nu=1}^{2 n}\left(s_{0}-s_{1}+s_{2}-s_{3}+\cdots\right) \sin \frac{\nu \pi}{2 N+1} .
\end{aligned}
$$

Proof. The identity (5.15) for $S_{N}(\omega)$ follows immediately from the definition (5.13) because, for $\nu=1, \cdots, 2 N$,

(5.16) $\sin \omega(\nu+(2 N+1) k) \Delta x=(-)^{k} \sin \nu \pi /(2 N+1) \quad(k=0,1, \cdots)$.

From the definition (5.13) for $C_{N}(\omega)$, we have

$$
C_{N}(\omega)=\frac{1}{2} \Delta x \sum_{\mu=-\infty}^{\infty} \psi\left(\left(\mu+\frac{1}{2}\right) \Delta x\right) \cos \omega\left(\mu+\frac{1}{2}\right) \Delta x .
$$

Letting $\mu=N-\nu(-\infty<\nu<\infty)$, we find

$$
\begin{aligned}
& C_{N}(\omega)= \frac{1}{2} \frac{\pi}{(2 N+1) \omega} \sum_{\nu=-\infty}^{\infty} \psi \\
&=\frac{\pi}{(2 N+1) \omega} \sum_{\nu=1}^{\infty} \frac{1}{\omega}\left\{\psi\left(\frac{\pi}{2}\left(\frac{1}{\omega}\left(\frac{\nu}{2 N+1}-\frac{1}{2}\right)\right)\right) \sin \frac{\nu \pi}{2 N+1}\right. \\
&\left.\quad-\psi\left(\frac{\pi}{\omega}\left(\frac{\nu}{2 N+1}+\frac{1}{2}\right)\right)\right\} \sin \frac{\nu \pi}{2 N+1} .
\end{aligned}
$$

The identity (5.15) for $C_{N}(\omega)$ now follows from (5.16).

We will now obtain convergent Euler expansions for the sums $\sum(-)^{k} \delta c_{k}$ and $\sum(-)^{k} s_{k}$. The sum $\sum(-)^{k} \delta c_{k}$ must be treated with special care. The first term, 
$\delta c_{0}$, involves some $\psi$ with negative arguments. In the extension of $\psi(x)$ as an even function for $-\infty<x<\infty$, discontinuities in the derivatives of $\psi$ may appear at $x=0$. For example, if $\psi(x)=\exp (-x)$ for $x \geqq 0$, the even extension exp $(-|x|)$ has discontinuous derivatives at the origin. To insure that the partial Euler sums behave well as $\omega \rightarrow \infty$, it is important to require that $\psi(x)$ have continuous derivatives only for $x \geqq 0$. Therefore, we will isolate the term $\delta c_{0}$. Thus, we will use the Euler expansion:

$$
\delta c_{0}+\sum_{k=1}^{\infty}(-)^{k} \delta c_{k}=\delta c_{\theta}-\sum_{n=1}^{\infty} 2^{-n} \delta^{n} c_{1} .
$$

For $\sum(-)^{k} s_{k}$ there is no such difficulty, and we simply write

$$
\sum_{k=0}^{\infty}(-)^{k} s_{k}=\sum_{n=0}^{\infty} 2^{-n-1} \delta^{n} s_{0} .
$$

The identities (5.18), (5.19) are direct applications of the Euler-Ames Theorem 5.1.

Now define the remainders $r_{m}$ and $\rho_{m}$ :

$$
\begin{array}{ll}
\sum_{n=1}^{\infty} 2^{-n} \delta^{n} c_{1}=\sum_{n=1}^{m-1} 2^{-n} \delta^{n} c_{1}+r_{m}(\omega) & (m \geqq 1), \\
\sum_{n=0}^{\infty} 2^{-n-1} s_{0}=\sum_{n=0}^{m-1} 2^{-n-1} \delta^{n} s_{0}+\rho_{m}(\omega) & (m \geqq 0),
\end{array}
$$

where $\sum_{n=1}^{0}=\sum_{n=0}^{-1}=0$. To obtain the behavior of $r_{m}(\omega)$ as $\omega \rightarrow \infty$, we use the representation

$$
r_{m}(\omega)=2^{-m+1} \sum_{k=0}^{\infty}(-)^{k} \delta^{m} c_{k+1} \quad(m \geqq 1)
$$

which follows from $(\tilde{5} . \overline{5})$. We can now use Lemma $\tilde{5} .1$ if we define

$$
r_{m}=2 R_{m}, \quad c_{k+1}=a_{k}, \quad \psi\left(\pi / \omega\left(\nu /(2 N+1)+x+\frac{1}{2}\right)\right)=a(x) .
$$

We have

$$
\left(\frac{d}{d x}\right)^{m+1} a(x)=\left(\frac{\pi}{\omega}\right)^{m+1} \psi^{(m+1)}\left(\frac{\pi}{\omega}\left(\frac{\nu}{2 N+1}+x+\frac{1}{2}\right)\right) .
$$

The inequality (5.6) of Lemma $\tilde{5} .1$ now yields

$$
\left|r_{m}\right| \leqq 2^{-m+1} \int_{0}^{\infty}\left(\frac{\pi}{\omega}\right)^{m+1}\left|\psi^{(m+1)}(\xi)\right| d x
$$

where $\xi=(\pi / \omega)\left((\nu /(2 N+1))+x+\frac{1}{2}\right)$. Now, for $\nu \geqq 1$ and $\omega>0$,

$$
\xi \geqq\left(\nu /(2 N+1)+\frac{1}{2}\right) \pi / \omega>0 \text { when } x \geqq 0 .
$$

Therefore, (5.24) gives

$$
\left|r_{m}\right| \leqq 2^{-m+1}\left(\frac{\pi}{\omega}\right)^{m} \int_{0}^{\infty}\left|\psi^{(m+1)}(\xi)\right| d \xi \quad(m \geqq 1) .
$$

To estimate $\rho_{m}(\omega)$ as $\omega \rightarrow \infty$, we use (5.5) to write

$$
\rho_{m}(\omega)=2^{-m} \sum_{k=0}^{\infty}(-)^{k} \delta^{m} s_{k} \quad(m \geqq 0) .
$$


To use Lemma 5.1 , we let

$$
\rho_{m}=R_{m}, \quad s_{k}=a_{k}, \quad \phi((\pi / \omega)[\nu /(2 N+1)+x]) .
$$

We then conclude

$$
\left|\rho_{m}\right| \leqq 2^{-m}\left(\frac{\pi}{\omega}\right)^{m} \int_{0}^{\infty}\left|\phi^{(m+1)}(\xi)\right| d \xi \quad(m \geqq 0) .
$$

We have thus proved the following theorem:

Theorem 5.2. For $x \geqq 0$ let $\psi(x)$ and (or) $\phi(x)$ have $m+1$ continuous derivatives, with

$$
\int_{0}^{\infty}\left|\psi^{(m+1)}(x)\right| d x<\infty, \quad \int_{0}^{\infty}\left|\phi^{(m+1)}(x)\right| d x<\infty .
$$

For $\nu=1,2, \cdots, 2 N$ and $k=0,1,2, \cdots$ define $c_{k}$ and $s_{k}$ by $(5.14)$, where $\psi(-x)=$ $\psi(x)$ in the definition of $c_{0}$. Let the series

$$
\sum_{k=0}^{\infty}(-)^{k} \delta c_{k} \quad \text { and } \quad \sum_{k=0}^{\infty}(-)^{k} s_{k}
$$

converge for all sufficiently large $\omega>0$. Let $m$ be fixed. Then as $\omega \rightarrow \infty$

$$
\begin{array}{ll}
\sum_{k=0}^{\infty}(-)^{k} \delta c_{k}=\delta c_{0}-\sum_{n=1}^{m-1} 2^{-n} \delta^{n} c_{1}+O\left(\omega^{-m}\right) & (m \geqq 1), \\
\sum_{k=0}^{\infty}(-)^{k} s_{k}=\sum_{n=0}^{m-1} 2^{-n-1} \delta^{n} s_{0}+O\left(\omega^{-m}\right) & (m \geqq 0) .
\end{array}
$$

If $m \rightarrow \infty$, for fixed $\omega$ we have convergence (5.18), (5.19).

These results give a numerical method for computing the integrals

$$
C(\omega)=\int_{0}^{\infty} \psi(x) \cos \omega x d x, \quad S(\omega)=\int_{0}^{\infty} \phi(x) \sin \omega x d x .
$$

First we approximate the integrals by trapezoidal sums $C_{N}(\omega), S_{N}(\omega)$; the errors $C_{N}-C$ and $S_{N}-S$ are given in formulas (4.28) and (4.29). In the forms (5.1j) the trapezoidal sums $C_{N}$ and $S_{N}$ involve infinite series $\sum(-)^{k} \delta c_{k}$ and $\sum(-)^{k} s_{k}$. These infinite series are approximated by truncated Euler series. For $m \geqq 1$ we find

$$
\begin{aligned}
& C_{N}(\omega)=\frac{\pi}{(2 N+1) \omega} \sum_{\nu=1}^{2 N}\left(\delta c_{0}-\sum_{\nu=1}^{m-1} 2^{-n} \delta^{n} c_{1}\right) \sin \frac{\nu \pi}{2 N+1}+O\left(\omega^{-m-1}\right), \\
& S_{N}(\omega)=\frac{\pi}{(2 N+1) \omega} \sum_{\nu=1}^{2 N} \sum_{n=0}^{m-1} 2^{-n-1} \delta^{n} s_{0} \sin \frac{\nu \pi}{2 N+1}+O\left(\omega^{-m-1}\right) .
\end{aligned}
$$

It is important to illustrate the power and the limitations of this technique. First we chose the example

$$
C(\omega)=\int_{0}^{\infty} e^{-x} \cos \omega x d x \quad(\omega>0) .
$$

Here we happen to know the integral explicitly:

$$
C(\omega)=1 /\left(1+\omega^{2}\right) .
$$


In this case we have $\psi(x)=\exp (-|x|)$, and (5.14) becomes

$$
\begin{aligned}
c_{k} & =\frac{1}{2} \exp \left\{-(\pi / \omega)\left|\nu /(2 N+1)+k-\frac{1}{2}\right|\right\} & & (k \geqq 0) ; \\
\delta c_{k} & =\frac{1}{2}\left(1-e^{-\pi / \omega}\right) \exp \left\{-(\pi / \omega)\left(\nu /(2 N+1)+k-\frac{1}{2}\right)\right\} & & (k \geqq 1) .
\end{aligned}
$$

The original series $\sum(-)^{k} \delta c_{k}$ appearing in $(5.15)$ is

$$
\delta c_{0}+\frac{1}{2}\left(1-e^{-\pi / \omega}\right)\left(\sum_{k=1}^{\infty}(-)^{k} \exp \left(\frac{-k \pi}{\omega}\right)\right) \exp \left\{-\frac{\pi}{\omega}\left(\frac{\nu}{2 N+1}-\frac{1}{2}\right)\right\} .
$$

This series converges very slowly for large $\omega$. To obtain the Euler series, we first compute

$$
\begin{aligned}
\delta^{n} c_{1} & =\frac{1}{2}\left(1-e^{-\pi / \omega}\right)^{n} \exp \left\{-(\pi / \omega)\left[\nu /(2 N+1)+\frac{1}{2}\right]\right\} \\
\delta c_{0} & =\frac{1}{2}\left[\exp \left(-\frac{\pi}{\omega}\left|\frac{\nu}{2 N+1}-\frac{1}{2}\right|\right)-\exp \left(-\frac{\pi}{\omega}\left(\frac{\nu}{2 N+1}+\frac{1}{2}\right)\right)\right] .
\end{aligned}
$$

The Euler partial sum in (5.33) now takes the form

$$
\delta c_{0}-\frac{1}{2} \exp \left\{-\frac{\pi}{\omega}\left(\frac{\nu}{2 N+1}+\frac{1}{2}\right)\right\} \sum_{n=0}^{m-1}\left(\frac{1-e^{-\pi / \omega}}{2}\right)^{n} .
$$

As $m \rightarrow \infty$ this series always converges faster than $\sum 2^{-n}$; and for fixed $m$, as $\omega \rightarrow \infty$, the remainder is $O\left(\omega^{-m}\right)$. An explicit upper bound for the remainder is given by $(5.25)$ :

$$
\left|r_{m}\right| \leqq 2^{-m+1}(\pi / \omega)^{m}
$$

This upper bound is useful for large $\omega$ but fails to demonstrate the convergence $r_{m} \rightarrow 0$ when $\omega \leqq \pi / 2$.

The following example shows that the Euler series should not usually be used when the theory of residues can be used. Consider the integral

$$
S(\omega)=\int_{0}^{\infty} \frac{x}{x^{2}+1} \sin \omega x d x
$$

The odd extension of $\phi(x)=x /\left(x^{2}+1\right)$ is, in this case, analytic along the whole line $-\infty<x<\infty$, including $x=0$. Using the theory of residues, one finds the familiar result

$$
S(\omega)=e^{-\omega} \quad(\omega>0) .
$$

Thus, $S(\omega) \rightarrow 0$ as $\omega \rightarrow \infty$ faster than any power $\omega^{-m-1}$. The asymptotic relation (5.33) for $S_{N}(\omega)$ still holds, but it gives a very weak result.

By contrast, consider

$$
C(\omega)=\int_{0}^{\infty} \frac{x}{x^{2}+1} \cos \omega x d x
$$

The even extension of $\psi(x)=x /\left(x^{2}+1\right)$ is not analytic at $x=0$. The theory of residues cannot be used, and in fact $C(\omega)$ does not tend exponentially to zero for $\omega \rightarrow \infty$. A few integrations by parts show that

$$
C(\omega)=-1 / \omega^{2}+O\left(1 / \omega^{4}\right) \text { as } \omega \rightarrow \infty .
$$


For the example (5.43) the Euler approximation (5.33) would be an adequate approximation to the trapezoidal sum $C_{N}$. The great error in computing this integral $C(\omega)$ would come in the approximation of $C$ by $C_{N}$. The first term in the series (4.28) for the error $C_{N}-C$ is

$$
-C((2 N+2) \omega)-C(2 N \omega) \cong 1 / 2 N^{2} \omega^{2} \text { as } N \rightarrow \infty .
$$

Since $C(\omega)$ does not tend rapidly to zero, it is important to use a fairly large number $N$.

The last example suggests the use of a correction term. After $N$ has been fixed, and after $C_{N}(\omega)$ or $S_{N}(\omega)$ has been computed from an Euler partial sum (5.33), additional accuracy can usually be obtained by computing the correction term

$$
\gamma_{N}=C_{N}(2 N \omega)+C_{N}((2 N+2) \omega)
$$

or, for the sine integral,

$$
\sigma_{N}=S_{N}(2 N \omega)-S_{N}((2 N+2) \omega) .
$$

The terms $\gamma_{N}$ and $\sigma_{N}$ are numerical approximations to the first terms in the errorseries (4.28), (4.29). The improved value for $C(\omega)$ will be $C_{N}+\gamma_{N}$; the improved value for $S(\omega)$ will be $S_{N}+\sigma_{N}$.

California Institute of Technology

Pasadena, California 91109

1. H. HuRwitz \& P. F. Zweifel, "Numerical quadrature of Fourier transform integrals," $M T A C$, v. 10,1956 , pp. 140-149. MR 18, 337.

2. L. A. SснмітTRoth, "Numerical inversion of Laplace transforms," Comm. ACM, v. 3, 1960, pp. 171-173. MR 22 * 7249.

3. L. N. G. Filon, "On a quadrature formula for trigonometic integrals," Proc. Roy. Soc. Edinburgh, v. 49,1928 , pp. 38-47.

4. Y. L. Luke, "On the computation of oscillatory integrals," Proc. Cambridge Philos. Soc., v. 50, 1954, pp. 269-277. MR 15, 992.

5. F. B. Hildebrand, Introduction to Numerical Analysis, McGraw-Hill, New York, 1956. MR 17, 788 .

6. KonRad Knopp, Theorie und Anwendung der Unendlichen Reihen, 4th ed., Springer, Berlin, 1947; English transl., Theory and Applications of Infinite Series, Blackie, London, 1949. MR 10, 446.

7. L. D. AMES, Ann. of Math. (2), v. 3, 1901, p. 185.

8. Y. L. LuKE, "Simple formulas for the evaluation of some higher transcendental functions," $J$. Wath. and Phys., v. 34, 1955, pp. 298-307. 